\title{
REPRESENTATIONS AND POSITIVE DEFINITE FUNCTIONS ON TOPOLOGICAL SEMIGROUPS
}

\author{
by J. W. BAKER and M. LASHKARIZADEH-BAMI
}

(Received 1 September, 1994)

\begin{abstract}
A number of theorems are established about positive definite functions and representations of certain topological semigroups. In particular we establish theorems which show that measurable positive definite functions and measurable representations can each be decomposed into the sum of two parts one of which is continuous and the other of which is "small".
\end{abstract}

1. Introduction. In this paper we aim to prove some theorems about positive definite functions, and about the closely related concept of representations, on locally compact topological semigroups; we shall also answer problems raised in [8] and [9] by improving previous theorems on these topics. Theorems about these concepts must, of necessity, apply to the special case of a topological group, and therefore will normally be a "generalization" of a known result for locally compact groups. The theorems we shall establish are true for groups, as a result of the existence of the group algebra, and so our results will often only apply to semigroups in which there is an analogue of the group algebra. Such semigroups are the so-called foundation semigroups; these semigroups form a large family but do not include all commonly met semigroups; the family includes all topological groups and all discrete semigroups, and is closed under products and subsemigroups and (under some restrictions) quotients.

Throughout this paper, we shall be concerned with a semigroup $S$ with a weight function $w$, and with functions and representations that are $w$-bounded. The original research which led to this paper was an attempt to prove the two main theorems of Section 4 , which concern the representations of positive definite functions and representations, respectively, as the sum of two of them, one being continuous and the other being "small", (4.6 and 4.9). In Section 3 we introduce a lemma of Stone-Weierstrass type that is an essential tool for the results of Section 4, and is also used in Section 6. Firstly, Theorem 4.2 provides a "Bochner theorem" for $w$-bounded, positive definite functions. We also establish Theorems 5.2 and 6.2 which establish necessary and sufficient conditions for the semi-simplicity of the algebra $M(S, w)$ and for the ${ }^{*}$-semi-simplicity of $M(S, w)$ if $S$ has an involution, respectively.

\section{Definitions and notations.}

2.1. Throughout this paper, except in Section $3, S$ will denote a locally compact, Hausdorff topological semigroup. We shall assume that there is a weight function $w$ on $S$. By this we mean that $w: S \rightarrow \mathbb{R}_{+}$with the properties that $w$ is Borel measurable and that both $w$ and $w^{-1}$ are locally bounded (i.e. bounded on compact subsets of $S$ ) and that $w(x y) \leqslant w(x) w(y)$ for $x, y \in S$. Further, if $S$ has an involution $*$ we shall assume that $w\left(x^{*}\right)=w(x)$ for $x \in S$. We denote by $M(S, w)$ the set of complex, regular, signed measures $\mu$ (not necessarily bounded), of the form $\mu=\mu_{1}-\mu_{2}+i\left(\mu_{3}-\mu_{4}\right)$, where $\mu_{i}$ is

Glasgow Math. J. 38 (1996) 99-111. 
a positive regular measure on $S$ with $w \in L^{1}\left(S, \mu_{i}\right)$ for $i=1,2,3,4$. Note that the elements of $M(S, w)$ are not measures in certain strict senses, in that $\mu(B)$, for a Borel set $B$, need not have meaning, but on the other hand $\mu(B)$ does have meaning in the case when $B$ is relatively compact. We leave it to the reader to check that nothing in this paper is affected by this sort of problem. We can define, for $f \in \bigcap_{i=1}^{4} L^{1}\left(S, \mu_{i}\right)$,

$$
\int_{S} f d \mu=\int_{S} f d \mu_{1}-\int_{S} f d \mu_{2}+i\left(\int f d \mu_{3}-\int f d \mu_{4}\right) .
$$

If $\mu \in M(S, w)$ we can define a bounded regular Borel measure $w \cdot \mu$ on $S$ by the equation

$$
\int f d(w . \mu)=\int f w d \mu \quad\left(f \in C_{b}(S)\right)
$$

since $f w$ is certainly in $\bigcap_{i=1}^{4} L^{1}\left(S, \mu_{i}\right)$. The map $\mu \mapsto w . \mu$ is clearly a linear bijection of $M(S, w)$ onto $M(S)$. So we can define $\|\mu\|_{w}=\|w . \mu\|$ for $\mu \in M(S, w)$, to make $M(S, w)$ into a Banach lattice (the norm of $w . \mu$ is the total variation norm). Finally, we make $M(S, w)$ into a Banach algebra by defining a convolution product by the rule

$$
(\mu * v)(f)=\int_{S} \int_{S} f(x y) d \mu(x) d v(y) \quad\left(\mu, v \in M(S, w), \quad f \in C_{00}(S)\right) .
$$

A simple adaptation of the proof of the Riesz representation theorem shows that $\mu * v$ is well-defined. By part (iii) of Theorem 4.6 of [8] we can conclude that (1) is still valid for any $w$-bounded, Borel measurable function $f$ on $S$. (We say that $f$ is $w$-bounded if there is $k \in \mathbb{R}$ such that $|f(x)| \leqslant k w(x)$ for all $x$ in $S$.)

Recall (see, for example, [1] or [4]) that $M_{a}(S)$ (or $\tilde{L}(S)$ ) denotes the set of all measures $\mu$ in $M(S)$ for which the mappings $x \mapsto \bar{x} *|\mu|$ and $x \mapsto|\mu| * \bar{x}$ from $S$ to $M(S)$ are weakly continuous. As in [8], we can define $M_{a}(S, w)$ (or $\tilde{L}(S, w)$ ) as the set of measures $\mu$ in $M(S, w)$ for which $w . \mu$ is in $M_{a}(S)$. Then $M_{a}(S, w)$ is a closed, two-sided $L$-ideal of $M(S, w)$. We call $S$ a foundation semigroup if $\cup\left\{\operatorname{supp}(\mu): \mu \in M_{a}(S)\right\}$ is dense in $S$.

2.2. We assume that the reader is familiar with the notions of representations of $*$-algebras and of *-semi-simplicity of Banach *-algebras. Most of the following definitions are to be found in [2], [8] or [9]. If $S$ is a topological semigroup with continuous involution $*$, a *-representation $V$ of $S$ by bounded operators on a Hilbert space $H$ is a homomorphism $x \mapsto V_{x}$ of $S$ into $B(H)$ such that $V_{x^{*}}=V_{x}^{*}$ for all $x \in S$. The representation $V$ is cyclic if there is a cyclic vector $\xi \in H$; i.e. an element $\xi \in H$ such that the span of $\left\{V_{x} \xi: x \in S\right\}$ is dense in $H$; it is $w$-bounded if there is a real number $k$ such that $\left\|V_{x}\right\| \leqslant k w(x)$ for all $x \in S$; and it is weakly continuous resp. Borel measurable, resp, $\mu$-measurable if the function $x \mapsto\left\langle V_{x} \xi, \eta\right\rangle$ is continuous (resp. Borel measurable, resp, $\mu$-measurable) for all $\xi, \eta \in H$. Observe that $V$ is $w$-bounded if and only if the function $x \mapsto\left\langle V_{x} \xi, \eta\right\rangle$ is $w$-bounded for all $\xi, \eta \in H$, and that the $*$-representation $V$ is $w$-bounded if and only if $\left\|V_{x}\right\| \leqslant w(x)$ for all $x \in S$. Finally, $R(S, w)$ denotes the set of all $w$-bounded 
continuous *-representations of the topological semigroup $S$ with involution, by bounded operators on Hilbert space.

If $M$ is a vector space of measures on $S$ then a function $f$ is said to be $M$-negligible if $|\mu|(\{x \in S: f(x) \neq 0\})=0$ for all $\mu \in M$. The representation $V$ is said to be $M$-negligible if $\left\{x:\left\langle V_{x} \xi, \eta\right\rangle \neq 0\right\}$ is $M$-negligible for all $\xi, \eta \in H$.

2.3. If $S$ is a semigroup with involution, then a complex-valued function $\varphi$ on $S$ is positive-definite if

$$
\sum_{i=1}^{n} \sum_{j=1}^{n} c_{i} \bar{c}_{j} \varphi\left(x_{i} x_{j}^{*}\right) \geqslant 0
$$

for all choices $\left\{x_{1}, x_{2}, \ldots, x_{n}\right\}$ from $S$ and $\left\{c_{1}, c_{2}, \ldots, c_{n}\right\}$ from $\mathbb{C}$. We denote by $\Gamma_{w}$ the set of all $w$-bounded and continuous semicharacters of $S$ (i.e. homomorphisms from $S$ to $\mathbb{C}$ which are not identically zero). If $S$ has an involution then $\Gamma_{n}^{*}$ is the set of continuous *-semicharacters (i.e. semicharacters which are $*$-homomorphisms). Clearly if $\chi \in \Gamma_{*}$, then $|\chi(x)| \leqslant w(x)(x \in S)$. We shall denote $\Gamma_{1}$ by $\hat{S}$.

We recall from [9, Theorem 2.10] that there is a one-one correspondence between $\Gamma_{w}$ and $M_{a}(S, w)$ that is defined as follows.

For $\chi \in \Gamma_{w}$, define a complex homomorphism $h$ on $M_{a}(S, w)$ by

$$
h(\mu)=\int_{S} \chi(x) d \mu(x) \quad\left(\mu \in M_{a}(S, w)\right)
$$

Further, $\chi$ is determined from $h$ by the rule

$$
\chi(x)=\frac{h(\mu * \bar{x})}{h(\mu)} \quad(x \in S)
$$

for any $\mu \in M_{a}(S, w)$ with $h(\mu) \neq 0$.

This correspondence is a homeomorphism if $M_{a}(S, w)$ has the Gelfand topology and $\Gamma_{w}$, the compact open topology; in particular $\Gamma_{n}$ is locally compact.

3. A lemma of Stone-Weierstrass type. The Stone-Weierstrass theorem tells us that if $X$ is a compact Hausdorff space then certain conditions on a subalgebra $A$ of $C(X)$ ensure that $A$ is dense in $C(X)$. It follows that if $\mu \in M(X)$ then such an $A$ is automatically dense in $L^{p}(X,|\mu|)$ for $p \geqslant 1$. Unfortunately, the theorem requires that $A$ contains the function 1 , and this may not be true in the applications to be used below. For that reason we require the following results.

Let $X$ be a locally compact, Hausdorff topological space. Let $C_{b}(X)$ denote the set of bounded and continuous complex-valued functions on $X$ and $C_{b}^{\mathbb{R}}(X)$ the set of real functions in $C_{b}(X)$. We give these spaces the supremum norm.

Lemma 3.1. Let $\mu \in M(X)$ (the bounded regular Borel measures on $X$ ) (with $\mu \neq 0$ ), and let $p \geqslant 1$. Let $A$ be a self-conjugate subalgebra of $C_{b}(X)$ such that and

(i) for each $x \in X$ there is $f \in A$ with $f(x) \neq 0$,

(ii) for $x, y \in X$ with $x \neq y$ there is $f \in A$ with $f(x) \neq f(y)$.

Then $A$ is dense in $L^{p}(X,|\mu|)$. 
Proof. We can assume that $\mu>0$. Let $f \in C_{b}^{R}(X)$. We shall show that for any $\varepsilon>0$ there is $q \in A$ with

$$
\int_{X}|f(x)-g(x)|^{p} d \mu(x)<\varepsilon
$$

The rest of the proof is then as in the proof of (31.4) of [7] (pp. 211-212, last paragraph). Take $\delta \in(0,1)$, to be determined later. Choose $K \subset X$ compact with $\mu(X \backslash K)<\delta$. The restriction of $A$ to $K$ is a subalgebra of $C(K)$. By the Stone-Weierstrass theorem (for example one can use the version given in $[11$, p. 166, Theorem A]) $A$ is dense in $C(K)$, and so we can find $g \in A$ with

$$
|g(x)-f(x)|<\delta \quad(x \in K) .
$$

Let $M=\max \{\|f\|,\|g\|\}$, and let $h$ be the continuous real-valued function on the interval $[-M-1, M+1]$ defined by

$$
h(t)= \begin{cases}t & \text { for }-\|f\|-1 \leqslant t \leqslant\|f\|+1, \\ \|f\|+1 & \text { for } \quad\|f\|+1<t \leqslant M+1, \\ -\|f\|-1 & \text { for }-M-1 \leqslant t<-\|f\|-1 .\end{cases}
$$

By the Weierstrass approximation theorem, we can find a polynomial $r$ such that $|r(t)-h(t)|<\delta$ for all $t \in[-M-1, M+1]$. Put $q(x)=r(g(x))$ for $x \in X$; then $q \in A$.

Now if $x \in K$, then

$$
|q(x)-f(x)| \leqslant|r(g(x))-g(x)|+|g(x)-f(x)|<2 \delta,
$$

since $|g(x)|<|f(x)|+\delta<\|f\|+1$. Also, if $x \in X \backslash K$ then

$$
\begin{aligned}
|q(x)-f(x)| \leqslant|q(x)|+|f(x)| & \leqslant|h(g(x))|+\delta+\|f\| \\
& \leqslant 2\|f\|+1+\delta .
\end{aligned}
$$

Hence

$$
\begin{aligned}
\int_{X}|q(x)-f(x)|^{p} d \mu(x) & =\int_{K}|q(x)-f(x)|^{p} d \mu(x)+\int_{X \backslash K}|q(x)-f(x)|^{p} d \mu(x) \\
& \leqslant(2 \delta)^{p} \mu(K)+\mu(X \backslash K)(2\|f\|+1+\delta)^{p} \\
& \leqslant(2 \delta)^{p}\|\mu\|+\delta(2\|f\|+1+\delta)^{p} .
\end{aligned}
$$

If we choose $\delta$ small enough, we get the required result.

Corollary 3.2. If $A$ is as in Lemma 3.1 and $\mu \in M(X)$ is such that $\mu(f)=0$, for all $f$ in $A$, then $\mu=0$.

Proof. Suppose that $\mu \neq 0$. Apply Lemma 3.1 with $p=1$. If $f \in C_{0}(X) \subseteq L^{1}(X,|\mu|)$ and $\varepsilon>0$ there is $h \in A$ with $\int_{S}|f(x)-h(x)| d|\mu|(x)<\varepsilon$. Therefore $\int_{X}|f(x)| d|\mu|(x)<\varepsilon$ and so $\mu=0$.

4. Decomposition of exponentially bounded representations and positive definite functions. The first result of this section answers question (b) of [9]. Theorems 4.6 and 4.9 provide our decomposition theorems. Examples 4.7 and 4.8 provide counter-examples 
to show that the decompositions are not trivial and that the introduction of foundation semigroups is necessary.

LEMMA 4.1. Let $S$ be any commutative topological semigroup with involution $*$ with a weight function $w$ such that $\Gamma_{w^{*}}^{*}$ is a locally compact semigroup in the compact-open copology (for example, $S$ could be a foundation semigroup with identity). If $\lambda \in M\left(\Gamma_{n}^{*}\right)$ and if $\int_{\Gamma_{*}^{*}} \chi(x) d \lambda(\chi)=0$, for all $x \in S$, then $\lambda=0$.

Proof. Let $A$ denote the subspace of $C_{b}\left(\Gamma_{w}^{*}\right)$ generated by the set $\{\hat{x}: x \in S\}$, where $\hat{x}(\chi)=\chi(x)$ for $\chi \in \Gamma^{*}$. Then $A$ satisfies the conditions of Corollary 3.2 , and $\int f(\chi) d \lambda(\chi)=0$ for all $f \in A$. Therefore $\lambda=0$.

For the rest of this section $S$ will be a commutative foundation semigroup with identity $e$, involution $*$, and weight function $w$. For $\mu \in M_{a}(S, w)$ define $\hat{\mu} \in C_{b}\left(\Gamma_{w}^{*}\right)$ by

$$
\hat{\mu}(\chi)=\int_{S} \chi(x) d \mu(x) \quad\left(\chi \in \Gamma_{u}^{*}\right) .
$$

We can immediately combine Lemma 4.1 with Theorem 2.12 of [9] to answer question (b) of [9].

THEOREM 4.2. (The Bochner theorem for exponentially bounded, continuous, positive definite functions on foundation semigroups). Let $S$ and $w$ be as above. A function $\varphi: S \rightarrow \mathbb{C}$ is $w$-bounded, continuous and positive definite if and only if there is a unique positive measure $\lambda_{\varphi} \in M\left(\Gamma_{w}^{*}\right)$ such that

$$
\varphi(x)=\int_{\Gamma_{u}^{*}} \chi(x) d \lambda_{\varphi}(\chi) \quad(x \in S) .
$$

Let $P(S, w)$ denote the set of all $w$-bounded and continuous positive-definite functions on $S$. Using Theorem 4.2 , for each $\lambda \in M\left(\Gamma_{w}^{*}\right)$ we define $\check{\lambda} \in P(S, w)$ by

$$
\check{\lambda}(x)=\int_{r_{n}^{*}} \chi(x) d \lambda(\chi) \quad(x \in S) .
$$

We then have the following corollary to Theorem 4.2. For the purposes of this result, let us define the vague topology on $M\left(\Gamma_{w}^{*}\right)$ as the weak topology induced by $C_{c}\left(\Gamma_{n}^{*}\right)$ and the constant functions on $\Gamma_{w^{*}}^{*}$ (This is a slightly different definition to that used by some authors.)

Corollary 4.3. Let $S$ and $w$ be as above, with $w \leqslant 1$. Then the map $\lambda \mapsto \grave{\lambda}$ of $M_{+}\left(\Gamma_{w}^{*}\right)$ onto $P(S, w)$ is a homeomorphism and isomorphism if $M_{+}\left(\Gamma_{w}^{*}\right)$ has the vague topology (defined as above) and $P(S, w)$ has the topology of uniform convergence on compact subsets of $S$.

Proof. By Theorem 4.2, the map $\lambda \mapsto \grave{\lambda}$ is a bijection. Let $\left(\lambda_{\alpha}\right)$ be a net in $M_{+}\left(\Gamma_{w}^{*}\right)$ and let $\lambda \in M_{+}\left(\Gamma_{w}^{*}\right)$. Suppose that $\check{\lambda}_{\alpha} \rightarrow \check{\lambda}$ in $P(S, w)$. By Theorem 2.4 of $[\mathbf{1 0}]$, this is equivalent to the two statements that

$$
\int \check{\lambda}_{\alpha}(x) d \mu(x) \rightarrow \int \check{\lambda}(x) d \mu(x)
$$


for all $\mu \in M_{a}(S, w)$, and

$$
\check{\lambda}_{\alpha}(e) \rightarrow \check{\lambda}(e)
$$

Now (3) says that $\lambda_{\alpha}\left(\Gamma_{w}^{*}\right) \rightarrow \lambda\left(\Gamma_{w}^{*}\right)$, that is (since the measures are positive) $\left\|\lambda_{\alpha}\right\| \rightarrow\|\lambda\|$. So we can suppose that the net $\left(\lambda_{\alpha}\right)$ is bounded.

Also, by Fubini's theorem, (2) says that

$$
\int_{\Gamma^{*}}\left(\int_{S} \chi(x) d \mu(x)\right) d \lambda_{\alpha}(\chi) \rightarrow \int_{\Gamma_{*}^{*}}\left(\int_{S} \chi(x) d \mu(x)\right) d \lambda(\chi) ;
$$

that is

$$
\int_{\Gamma^{*}} \hat{\mu}(\chi) d \lambda_{\alpha}(\chi) \rightarrow \int_{\Gamma^{*}} \hat{\mu}(\chi) d \lambda(\chi)
$$

for all $\mu \in M_{a}(S, w)$.

Now $\left.\left\{\hat{\mu}: \mu \in M_{a}(S, w)\right)\right\}$ is a closed, separating and self-conjugate subalgebra of $C_{0}\left(\Gamma_{w}^{*}\right)$ that vanishes identically at no point of $\Gamma_{w}^{*}$, and so is uniformly dense in $C_{0}(S, w)$, by the Stone-Weierstrass theorem. Hence conditions (2) and (3) imply that

$$
\int_{\Gamma_{*}^{*}} f(\chi) d \lambda_{\alpha}(\chi) \rightarrow \int_{\Gamma_{*}^{*}} f(\chi) d \lambda(\chi)
$$

for all $f \in C_{0}\left(\Gamma_{w}^{*}\right)$, and so $\lambda_{\alpha} \rightarrow \lambda$ in the vague topology. The converse is also clear from the above argument.

Finally it is clear that $(\mu * \lambda)^{\check{ }}=\check{\mu} \check{\lambda}$ for $\mu, \lambda$ in $M\left(\Gamma_{w}^{*}\right)$.

We can now give the first of two results which enable us to construct *-representations.

Proposition 4.4. Let $S$ and $w$ be as above. Let $\lambda$ be a positive measure in $M\left(\Gamma_{w}^{*}\right)$. For each $x \in S$ and $f \in L^{2}\left(\Gamma_{w}^{*}, \lambda\right)$ define $U_{x}^{\lambda}(f)=\hat{x} f$, where $\hat{x}(\chi)=\chi(x)$ for $\chi \in \Gamma_{w^{*}}^{*}$ Then $U^{\lambda}$ defines a w-bounded, continuous, cyclic *-representation of $S$ on $L^{2}\left(\Gamma_{w}^{*}, \lambda\right)$.

Proof. The case $\lambda=0$ is trivial, and so assume that $\lambda>0$. All that is non-trivial is to show that $U^{\lambda}$ is continuous and cyclic. We fix $x_{0} \in S$, and take $\varepsilon>0$. Choose a non-zero $g$ in $L^{2}\left(\Gamma_{w}^{*}, \lambda\right)$. Let $G$ be a relatively compact neighbourhood of $x_{0}$ in $S$, and choose $K$ such that $w(x) \leqslant K$ for $x \in G$. Now find a compact subset $F$ of $\Gamma_{w}^{*}$ such that

$$
\int_{\Gamma_{w}^{*} \backslash F}|g(\chi)|^{2} d \lambda(\chi)<\frac{\varepsilon}{8 K^{2}}
$$


Then $H=\left\{x \in S:\left|\chi(x)-\chi\left(x_{0}\right)\right|<\frac{\varepsilon}{2\|g\|^{2}}\right.$ for all $\left.\chi \in F\right\}$ is a neighbourhood of $x_{0}$ in $S$. If $x \in G \cap H$ then

$$
\begin{aligned}
\left\|U_{x}^{\lambda}(g)-U_{x_{0}}^{\lambda}(g)\right\|^{2}= & \int_{F}\left|\chi(x)-\chi\left(x_{0}\right)\right|^{2}|g(\chi)|^{2} d \lambda(\chi)+\int_{\Gamma_{*}^{*} \vee}\left|\chi(x)-\chi\left(x_{0}\right)\right|^{2}|g(\chi)|^{2} d \lambda(\chi) \\
& <\frac{\varepsilon}{2\|g\|^{2}} \int_{F}|g(\chi)|^{2} d \lambda(\chi)+4 K^{2} \int_{\Gamma_{*}^{*} \vee F}|g(\chi)|^{2} d \lambda(\chi) \\
& <\frac{\varepsilon}{2}+\frac{\varepsilon}{2}=\varepsilon .
\end{aligned}
$$

Therefore $U^{\lambda}$ is (strongly) continuous.

Now, by Lemma 3.1 the linear span of $\{\hat{x}: x \in S\}$ is dense in $L^{2}\left(\Gamma_{w}^{*}, \lambda\right)$, so that the function 1 is a cyclic vector for $U^{\lambda}$.

Proposition 4.5. Let $S$ and $w$ be as above. Let $\lambda$ be a positive measure in $M\left(\Gamma_{w}^{*}\right)$. For each $\mu \in M_{a}(S, w)$ and each $f \in L^{2}\left(\Gamma_{w}^{*}, \lambda\right)$ define $V_{\mu}(f)=\hat{\mu} f$. Then the mapping $\mu \mapsto V_{\mu}$ is a cyclic *-representation of the Banach *-algebra $M_{a}(S, w)$ by bounded operators on $L^{2}\left(\Gamma_{w}^{*}, \lambda\right)$.

Proof. The involution on $M_{a}(S, w)$ is given by the formula

$$
\int_{S} f(x) d \mu^{*}(x)=\overline{\int_{S} f\left(x^{*}\right) d \mu(x)} \quad\left(f \in C_{00}(S), \mu \in M_{a}(S, w)\right) .
$$

For this involution $\left(\mu^{*}\right)^{\wedge}=\overline{\hat{\mu}}$, for each $\mu$ in $M_{a}(S, w)$. It is easy to check that $\mu \mapsto V_{\mu}$ is a bounded *-representation of $M_{a}(S, w)$. As in the previous proof, we show that 1 is a cyclic vector for the representation. Let $A$ denote the liner span in $C_{0}\left(\Gamma_{w}^{*}\right)$ of $\left\{\hat{\mu}: \mu \in M_{a}(S, w)\right\}$. Then $A$ separates the points of $\Gamma_{w}^{*}$, is self-conjugate and vanishes identically at no point of $\Gamma_{w^{*}}^{*}$ Hence $A$ is dense in $L^{2}\left(\Gamma_{w}^{*}, \lambda\right)$ (for example, by Lemma 3.1). The result now follows.

We now state our decomposition theorem for $w$-bounded positive definite functions.

THEOREM 4.6. Let $S$ and $w$ be as above. Let $\varphi$ be $\mu$-measurable for all $\mu \in M_{a}(S, w)$ and $a w$-bounded positive definite function on $S$. Then there are unique $w$-bounded positive definite functions $\varphi_{1}$ and $\varphi_{2}$ on $S$ such that $\varphi=\varphi_{1}+\varphi_{2}$, where $\varphi_{1}$ is continuous and $\varphi_{2}$ vanishes $\mu$ almost everywhere, for all $\mu$ in $M_{a}(S, w)$.

Proof. For each $s \in S$ define $\varphi_{s}: S \rightarrow \mathbb{C}$ by $\varphi_{s}(t)=\varphi\left(s^{*} t\right)(t \in S)$. Let $H_{0}$ denote the linear subspace of $\mathbb{C}^{S}$ (the space of all complex functions on $S$ ) generated by $\left\{\varphi_{s}: s \in S\right\}$. We make $H_{0}$ a pre-Hilbert space by defining

$$
\left\langle\varphi_{s}, \varphi_{t}\right\rangle=\varphi\left(s^{*} t\right) \quad(s, t \in S) .
$$

Theorem 4.1.14 of [3] shows that the mapping $V: S \rightarrow B\left(H_{0}\right)$, given by $V_{s}\left(\varphi_{l}\right)=\varphi_{s t}$ for $s, t \in S$, is a $w$-bounded, cyclic, *-representation of $S$ by bounded operators on $H_{0}$. Indeed $\left\|V_{s}\right\| \leqslant w(s)$, for all $s \in S$. It is also clear that $V$ is weakly $M_{a}(S, w)$-measurable.

Let $H$ denote the completion of $H_{0}$. Then $V$ can be extended uniquely to a 
$w$-bounded weakly $M_{a}(S, w)$-measurable cyclic *-representation of $S$ by bounded operators on $H$, with $\varphi$ as the cyclic vector. For simplicity, we also denote this extension by $V$.

By Theorem 5.2 of $[8]$, the formula

$$
\left\langle T_{\mu} \xi, \eta\right\rangle=\int_{S}\left\langle V_{x} \xi, \eta\right\rangle d \mu(x) \quad\left(\xi, \eta \in H, \mu \in M_{a}(S, w)\right),
$$

defines a bounded representation $T$ of $M_{a}(S, w)$ by bounded operators on $H$. Furthermore $V_{x}\left(T_{\mu}\right)=T_{\bar{x} * \mu}$ for $x \in S, \mu \in M_{a}(S, w)$. For fixed $\mu \in M_{a}(S, w)$ and $\xi, \eta \in H$ we claim that the function

$$
x \mapsto\left\langle V_{x} T_{\mu} \xi, \eta\right\rangle
$$

from $S$ to $\mathbb{C}$ is continuous. For let $\left(x_{\alpha}\right)$ be a net which converges to $x$ in $S$. Then

$$
\begin{aligned}
\left|\left\langle V_{x_{\mathrm{a}}} T_{\mu} \xi, \eta\right\rangle-\left\langle V_{x} T_{\mu} \xi, \eta\right\rangle\right| & =\left|\left\langle T_{x_{\mathrm{a}} * \mu-x * \mu} \xi, \eta\right\rangle\right| \\
& \leqslant\left\|\bar{x}_{\alpha} * \mu-\bar{x} * \mu\right\|_{w}\|\xi\|\|\eta\| \rightarrow 0
\end{aligned}
$$

by Proposition 4.7 of $[8]$. Let $M_{1}$ denote the closure of the linear span of $\left\{T_{\mu} \xi: \mu \in\right.$ $\left.M_{a}(S, w), \xi \in H\right\}$ in $H$. Then the function $x \mapsto\left\langle V_{x} \zeta, \eta\right\rangle$ is continuous for every $\zeta \in M_{1}$ and every $\eta \in H$. Now this implies that $V_{x}\left(M_{1}\right) \subseteq M_{1}$ for all $x \in S$, and so $M_{1}$ is an invariant subspace for $V$. Let $M_{2}$ be the orthogonal complement of $M_{1}$ in $H$. Since $V$ is a *-representation, $M_{2}$ is also an invariant subspace for $V$. Now if $\mu \in M_{a}(S, w), \xi \in H$, $\eta \in M_{2}$, then $T_{\mu} \xi \in M_{1}$ so that $\left\langle T_{\mu} \xi, \eta\right\rangle=0$. In particular, $\left\langle T_{\mu} \eta, \eta\right\rangle=0$ for all $\mu \in$ $M_{a}(S, w), \eta \in M_{2}$. So by (4) and Lemma 4.8 of $[8],\left\langle V_{x} \eta, \eta\right\rangle=0, \mu$ almost-everywhere for every $\mu \in M_{a}(S, w)$. Choose $\xi \in H$ such that $\varphi(x)=\left\langle V_{x} \xi, \xi\right\rangle(x \in S)$. (In other words $\xi$ is the image of $\varphi=\varphi_{e}$ under the embedding of $H_{0}$ in $H$.) Write $\xi=\xi_{1}+\xi_{2}$ with $\xi_{1} \in M_{1}$ and $\xi_{2} \in M_{2}$. Then

$$
\left\langle V_{x} \xi, \xi\right\rangle=\left\langle V_{x} \xi_{1}, \xi_{1}\right\rangle+\left\langle V_{x} \xi_{2}, \xi_{2}\right\rangle \quad(x \in S),
$$

so that $\varphi(x)=\varphi_{1}(x)+\varphi_{2}(x)$, where $\varphi_{1}(x)=\left\langle V_{x} \xi_{1}, \xi_{1}\right\rangle$ and $\varphi_{2}(x)=\left\langle V_{x} \xi_{2}, \xi_{2}\right\rangle$, for $x \in S$. It is now clear that $\varphi_{1}$ and $\varphi_{2}$ satisfy the desired conditions. The uniqueness of $\varphi_{1}$ and $\varphi_{2}$ follows from Lemma 4.8 of $[\mathbf{8}]$.

EXAmple 4.7. In this example, $w$ will be the function 1. Consider the non-foundation topological semigroup $S=([0,1], \min )$. By Proposition 4.4 .18 of [3], since every positive definite function on $S$ is non-negative and increasing, and since $M_{a}(S, w)=\{0\}$, the decomposition theorem above does not have meaning for $S$, in that many representations $\varphi=\varphi_{1}+\varphi_{2}$ will have $\varphi_{2}$ small in any meaningful sense.

Example 4.8. Again we take $w$ to be the function 1 . Let $S$ be the foundation topological semigroup $S=([0, x),+)$. The function $\varphi$ defined on $S$ by

$$
\varphi(x)= \begin{cases}0 & \text { if } x>0 \\ 1 & \text { if } x=0\end{cases}
$$

is an $M_{a}(S, w)$ measurable $w$-bounded positive definite function on $S$. Clearly $\varphi$ is not continuous, and so the decomposition $\varphi=\varphi_{1}+\varphi_{2}$ of Theorem 4.6 has $\varphi_{2} \neq 0$. The general form of the decomposition of an arbitrary positive definite function $\varphi$ on this semigroup is described in [3, Proposition 4.2, p. 113]. 
It might appear to the reader that this example is rather degenerate in that the support of $\varphi_{2}$ is only one point. We are not aware of any examples which are significantly different from this one. However, one can use the simple trick of taking the product of any locally compact abelian group $G$ with this semigroup, and then $\varphi_{2}$ will be the function given by

$$
\varphi_{2}(x, y)= \begin{cases}0 & \text { if } y>0 \\ p(x) & \text { if } y=0\end{cases}
$$

for $(x, y) \in G \times S$, where $p$ is a (continuous) positive definite function on $G$. We have been unable to decide whether there is a positive definite function on a foundation semigroup which is discontinuous at a dense set of points.

We now obtain a result parallel to Theorem 4.6 for representations.

THEOREM 4.9. Let $S$ and $w$ be as above. Let $V$ be a cyclic, w-bounded and $M_{a}(S, w)$ measurable *-representation of $S$ by bounded operators on the Hilbert space $H$. Then we can write $V$ as the direct sum $V^{\prime} \oplus V^{\prime \prime}$ of two such representations $V^{\prime}$ and $V^{\prime \prime}$, where $V^{\prime}$ is weakly continuous and is equivalent to some $U^{\lambda}$ for $\lambda \in M\left(\Gamma_{w}^{*}\right)$ (as in Proposition 4.4) and $V^{\prime \prime}$ is $M_{a}(S, w)$-negligible. Moreover, if $V$ is such that for every non-zero $\xi$ in $H$ the set $\left\{x \in S:\left\langle V_{x} \xi, \xi\right\rangle \neq 0\right\}$ is not $M_{a}(S, w)$-negligible then $V^{\prime \prime}=0$, and so $V$ is weakly continuous.

Proof. Let $\xi$ be a cyclic vector for $V$. Then the function $\varphi: S \rightarrow \mathbb{C}$ defined by $\varphi(x)=\left\langle V_{x} \xi, \xi\right\rangle$ is a $w$-bounded, $M_{a}(S, w)$-measurable and positive definite function on $S$. Write $\varphi=\varphi_{1}+\varphi_{2}$ as in Theorem 4.6. As in the proof of Theorem 4.6, we can write $H=M_{1} \oplus M_{2}$ and find $\xi_{1} \in M_{1}, \xi_{2} \in M_{2}$ so that $M_{1}$ and $M_{2}$ are complementary invariant subspaces and $\left\langle V_{x} \xi, \xi\right\rangle=\left\langle V_{x} \xi_{1}, \xi_{1}\right\rangle+\left\langle V_{x} \xi_{2}, \xi_{2}\right\rangle$, where $\left\langle V_{x} \xi_{1}, \xi_{1}\right\rangle=\varphi_{1}(x),\left\langle V_{x} \xi_{2}, \xi 2\right\rangle=\varphi_{2}(x)$ for $x \in S$. By Theorem 4.2, there is a positive measure $\lambda \in M\left(\Gamma_{w}^{*}\right)$ such that $\left\langle V_{x} \xi_{1}, \xi_{2}\right\rangle=$ $\int_{\Gamma_{i}} \chi(x) d \lambda(x)$ for $x \in S$. Let $V^{\prime}$ and $V^{\prime \prime}$ denote the restrictions of $V$ to $M_{1}$ and $M_{2}$ respectively. Now we can write $\left\langle V_{x} \xi_{1}, \xi_{1}\right\rangle=\left\langle U_{x}^{\lambda} 1,1\right\rangle$ for $x \in S$.

By Proposition 4.4, $U^{\lambda}$ is a cyclic representation with cyclic vector 1 and $V^{\prime}$ has cyclic vector $\xi_{1}$, and so by Proposition 2.4 of $[2], V^{\prime}$ is equivalent to $U^{\lambda}$. That $V^{\prime \prime}$ is $M_{a}(S, w)$-negligible is now clear, and then the final part of the statement of the theorem is also clear since $\xi_{2}$ must be zero.

5. The semisimplicity of the algebra $M(S, w)$. In this section we give a complete answer to question (a) of [9] by proving the following result.

THEOREM 5.1. Let $S$ be a commutative foundation topological semigroup with identity. Let $w$ be a weight function on $S$. Consider the following conditions.

(i) The commutative Banach algebra $M(S, w)$ is semisimple.

(ii) The commutative Banach algebra $M_{a}(S, w)$ is semisimple.

(iii) $\Gamma_{w}$ separates the points of $S$.

(iv) $\hat{S}$ separates the points of $S$.

(v) The commutative Banach algebra $M(S)$ is semisimple.

The first three conditions are equivalent and so are the last two, and the first three imply the last two.

Before proceeding to a proof of Theorem 5.1 we should make some remarks. The question posed in [9] was meant to ask whether conditions (i), (ii) and (iii) of Theorem 
5.1 are equivalent, which is what is answered. It is simple to see that (iii) and (iv) are not equivalent. For example, let $S$ be the additive semigroup of non-negative real numbers in its usual topology and let the weight function be $w(t)=e^{-t^{2}}$. Then clearly $\hat{S}$ separates the points of $S$, but $\Gamma_{w}$ is empty.

Proof. Using Theorem 4.2 of [9] we see that all we need to show is that (iii) implies (i).

First suppose that $S$ has a zero element 0 . Then $w(0)=w(x, 0) \leqslant w(x) w(0)$ so that $w(x) \geqslant 1$ for each $x \in S$. So in this case the constant function 1 is an element of $\Gamma_{w}$. However, it is shown in Theorem 4.2 of [9] that (iii) does imply (i) whenever there is $\chi \in \Gamma_{n^{\prime}}$ which is non-zero throughout $S$. So (iii) does imply (i) in this case.

Now suppose that $S$ does not have a zero-element. If $\Gamma_{n}$ separates the points of $S$, and if $z \in S$ is such that $\chi(z)=0$ for all $\chi \in \Gamma_{w}$ then $\chi(z x)=0=\chi(z)$ for all $x \in S$, so that $z$ is a zero element for $S$. Hence, for any $y \in S$ we can find $\chi \in \Gamma_{u^{\prime}}$ with $x(y) \neq 0$.

Let $\chi \in \Gamma_{w}$, and put $I=\{x \in S: \chi(x)=0\}$. Then we can write

$$
M(S, w)=M(I, w) \oplus M(S \backslash I, w)
$$

as a direct sum of an ideal and a subalgebra of $M(S, w)$, and so if $\mu \in M(S, w)$ we have $\mu=\left.\mu\right|_{I}+\left.\mu\right|_{S \checkmark}$. Clearly, the $w$-bounded and continuous semicharacters of $S \backslash I$ separate the points of the semigroup $S \backslash I$, and $\chi$ is non-zero throughout $S \backslash I$. Hence by what we have quoted above from Theorem 4.2 of [9], we see that $M(S \backslash I, w)$ is semisimple.

Now let $\mu \in M(S, w)$. If $\left.\mu\right|_{S \backslash} \neq 0$, then we can find a complex homomorphism $h_{0}$ on $M(S \backslash I, w)$ with $h_{0}(\mu) \neq 0$. Define a complex homomorphism $h$ on $M(S, w)$ by

$$
h(v)=h_{0}\left(\left.v\right|_{S \backslash V}\right) \quad(v \in M(S, w))
$$

Clearly $h(\mu) \neq 0$.

Now, given $\mu \in M(S, w)$ with $\mu \neq 0$, choose $x \in \operatorname{supp}(\mu)$, and $\chi \in \Gamma_{w}$ with $\chi(x) \neq 0$. Then $\left.\mu\right|_{S \backslash \backslash} \neq 0$, where $l$ is as above. Hence we can find a complex homomorphism $h$ of $M(S, w)$ with $h(\mu) \neq 0$ and so $M(S, w)$ is semisimple.

6. The $*$-semisimplicity of the algebra $M(S, w)$. In this section we show that a conjecture made by the second author in $[8]$ is true for the case $w \leqslant 1$. We first recall that if $V$ and $V^{\prime}$ are representations of a semigroup by bounded operators on the Hilbert spaces $H$ and $H^{\prime}$ respectively, then the tensor product $V \otimes V^{\prime}$ of $V$ and $V^{\prime}$ on $H \otimes H^{\prime}$, the tensor product of $H$ and $H^{\prime}$, is defined by the identity

$$
\left(V \otimes V^{\prime}\right)_{x}=V_{x} \otimes V_{x}^{\prime}, \text { for each } x \in S .
$$

Therefore

$$
\left\langle\left(V \otimes V^{\prime}\right)_{x}\left(\xi \otimes \xi^{\prime}\right), \eta \otimes \eta^{\prime}\right\rangle=\left\langle V_{x} \xi, \eta\right\rangle\left\langle V_{x}^{\prime} \xi^{\prime}, \eta^{\prime}\right\rangle
$$

for $x \in S, \xi, \eta \in H, \xi^{\prime}, \eta^{\prime} \in H^{\prime}$.

LeMma 6.1. Let $S$ be a topological semigroup with involution * (not necessarily commutative). Let $w$ be a weight function on $S$ with $w\left(x^{*}\right)=w(x) \leqslant 1$ for $x \in S$. If $R(S, w)$ separates the points of $S$, then the Banach *-algebra $M(S, w)$ is *-semisimple.

Proof. For each $V \in R(S, w)$, let $H_{V}$ denote the Hilbert space associated with $V$. For 
every $V, V^{\prime} \in R\left(S, w^{\prime}\right)$, we have $\left\|V_{x} \otimes V_{x}^{\prime}\right\| \leqslant\left\|V_{x}\right\|\left\|V_{x}^{\prime}\right\|$ for $x \in S$ by Theorem D.15 of [7]. Since $w \leqslant 1$ it follows that $V \otimes V^{\prime} \in R(S, w)$. Let $A$ denote the linear span of the set of functions of the form $x \mapsto\left\langle V_{x} \xi, \xi\right\rangle$ with $V \in R(S, w)$ and $\xi \in H_{V}$. Then $A$ is a selfconjugate subalgebra of $C_{b}(S)$. Since $R(S, w)$ separates the points of $S$, the algebra $A$ separates the points of $S$. We claim that for every $x \in S$ there is $f \in A$ with $f(x) \neq 0$. For suppose that $z \in S$ is such that $V_{z}=0$ for every $w$-bounded $*$-representation $V$ of $S$. Then $V_{x z}=V_{z x}=V_{z}=0$ and so $x z=z x=z$ for every $x \in S$. This implies that $z$ is a zero element for $S$. However, as in the proof of Theorem 5.1,w $\chi \equiv 1$ defines an element of $R(S, w)$-a contradiction. Hence there is $V$ with $V_{z} \neq 0$, and so $f \in A$ with $f(z) \neq 0$.

Let $v$ be a non-zero element of $M(S, w)$. Then $\mu=w . v$ is in $M(S)$, and clearly $|\mu|=w .|v|$. We claim that $A$ is dense in $L^{\prime}(S,|v|)$. Now, by Corollary $3.2, A$ is dense in $L^{1}(S,|\mu|)$. The Banach space dual of $L^{1}(S,|v|)$ is $L^{x}(S,|v|)$ (equivalence classes of bounded functions) in the usual way. Let $\varphi \in L^{x}(S,|v|)$ and suppose that

$$
\int_{S} \varphi(x) f(x) d|v|(x)=0
$$

for all $f \in A$. Let $f_{0} \in A$ be fixed. Then

$$
\int_{S} \varphi(x) f(x) f_{0}(x) d|v|(x)=0 .
$$

for all $f \in A$; that is

$$
\int_{S}\left[\varphi(x) \frac{f_{0}(x)}{w(x)}\right] f(x) d|\mu|(x)=0,
$$

for all $f \in A$. However, $\varphi f_{0} / w$ is a function bounded on $S$. Therefore

$$
\frac{\varphi(x) f_{0}(x)}{w(x)}=0 \quad(\mu \text { a.e. })
$$

and so $|\mu|\left(\{x \in S: \varphi(x) \neq 0\} \cap\left\{x \in S: f_{0}(x) \neq 0\right\}\right)=0$ for all $f_{0} \in A$. But the open sets $\left\{x \in S: f_{0}(x) \neq 0\right\}$, as $f_{0}$ varies over $R(S, w)$, cover $S$, by what we have shown above. Therefore $|\mu|\{x \in S: \varphi(x) \neq 0\}=0$, and so $\varphi=0, \mu$ a.e. However $|\mu|$ and $|v|$ have the same null sets and so clearly $A$ is dense in $L^{1}(S,|v|)$ since the only continuous linear functional on $L^{1}(S,|v|)$ that vanishes on $A$ is zero.

To show that $M(S, w)$ is semisimple, let $v \in M(S, w)$ be such that every bounded *-representation $T$ of $M(S, w)$ has $T_{v}=0$. Then, by Theorem 5.2 of [8],

$$
\int_{S}\left\langle V_{x} \xi, \eta\right\rangle d v(x)=0
$$

for all $w$-bounded Borel measurable *-representations $V$ of $S$. Therefore

$$
\int_{S} f(x) d v(x)=0
$$


for all $f \in A$, and so, since $A$ is dense in $L^{1}(S,|v|)$, for all $f \in L^{1}(S,|v|)$. In particular, choose $f$ of modulus 1 such that $|v|=f$. $v$. Then $f \in L^{1}(S,|v|)$, and so

$$
\int_{S} 1 d|v|(x)=0
$$

so that $v=0$. Therefore $M(S, w)$ is semisimple.

Combining this result with Theorem 5.4 of [8] we obtain the following result.

THEOREM 6.2. Let $S$ be a foundation topological semigroup with involution * (not necessarily commutative). Let $w$ be a weight function on $S$ with $w(x)=w\left(x^{*}\right) \leqslant 1$ for $x \in S$. Suppose that $S$ contains no zero element. The following conditions are equivalent.

(i) The Banach *-algebra $M(S, w)$ is *-semisimple.

(ii) The Banach *-algebra $M_{a}(S, w)$ is *-semisimple.

(iii) $R(S, w)$ separates the points of $S$.

REMARK. In contrast to the situation for commutative semigroups, the hypothesis that $w$ is bounded seems to be essential to our method of proof. Let $A$ be as in the proof of Lemma 6.1. In general $A$ is not an algebra; all we know is that if $f$ is in the linear span of all functions of the form $\left\langle V_{x}, \xi, \xi\right\rangle$ with $V \in R(S, 1), \xi \in H_{V}$, and $g \in A$ then $f . g \in R(S, w)$, which only tells us that $A$ is an algebra if $w \leqslant 1$. From this fact it is possible to prove that if $R(S)$ separates the points of $S$ and there is $f \in R(S, w)$ such that $f(x)>0$ for all $x \in S$ then $M(S, w)$ is semisimple without any conditions on $w$, but the argument does not seem to lead to a proof that (iii) implies (i) in Theorem 6.2. In the commutative case we were able to avoid this problem by using the trick in the proof of Theorem 4.2 of [9] which makes it possible to construct elements of $\hat{S}$ from elements of $\Gamma_{w}$. We have not found any similar trick for the non-commutative case. However it appears to the authors that semigroups and algebras with weights satisfying $w(x) \leqslant 1$ are probably of more interest that those for which $w(x) \geqslant 1$.

\section{REFERENCES}

1. A. C. Baker and J. W. Baker, Algebras of measures on a locally compact semigroup II, J. London Math. Soc. 2 (1970), 651-659.

2. J. W. Baker and M. Lashkarizadeh-Bami, On the representations of certain idempotent topological semigroups, Semigroup Forum 44 (1992), 245-254.

3. C. Berg, J. P. R. Christensen and P. Ressel, Harmonic analysis on semigroups (SpringerVerlag, New York, 1984).

4. H. A. M. Dzinotyiweyi, The analogue of the group algebra for topological semigroups (Pitman, 1984).

5. K. H. Hofmann, J. D. Lawson and J. S. Pym, The analytical and topological theory of semigroups (de Gruyter, 1990).

6. E. Hewitt and K. A. Ross, Abstract harmonic analysis, I (Springer-Verlag, 1963).

7. E. Hewitt and K. A. Ross, Abstract harmonic analysis, II (Springer-Verlag, 1970).

8. M. Lashkarizadeh-Bami, Representations of foundation semigroups and their algebras, Canadian J. Math. 37 (1985), 29-47.

9. M. Lashkarizadeh-Bami, Bochner's theorem and the Hausdorff moment theorem on foundation semigroups, Canadian J. Math. 37 (1985), 785-809. 
10. M. Lashkarizadeh-Bami, On various types of convergence of positive-definite functions on foundation semigroups, Math. Proc. Camb. Phil. Soc. 111 (1992), 325-330.

11. G. F. Simmons, Introduction to topology and modern analysis (McGraw-Hill, 1963).

Department of Pure Mathematics

THE UNIVERSITY

SHEFFIELD S3 7RH

ENGLAND, U.K.
Department of Mathematics UNIVERSITY OF ISFAHAN

ISFAHAN

IRAN 